\title{
The No-Arbitrage Property under a Change of Numéraire
}

\author{
FREDDY DELBAEN \\ Department of Mathematics \\ VRIJe Universiteit Brussel \\ PleinlaAN 2 \\ B-1050 BRUsSELS \\ BELGIUM \\ WALTER SCHACHERMAYER \\ INSTITUT FÜR STATISTIK \\ UNIVERSITÄT WIEN \\ BRÜNNERSTRASSE 72 \\ A-1210 WIEN \\ Austria
}

\begin{abstract}
For a price process that has an equivalent risk neutral measure, we investigate if the same property holds when the numéraire is changed. We give necessary and sufficient conditions under which the price process of a particular asset - which should be thought of as a different currency - can be chosen as new numéraire. The result is related to the characterization of attainable claims that can be hedged. Roughly speaking: the asset representing the new currency is a reasonable investment (in terms of the old currency) if and only if the market does not permit arbitrage opportunities in terms of the new currency as numéraire. This rough but economically meaningful idea is given a precise content in this paper. The main ingredients are a duality relation as well as a result on maximal elements. The paper also generalizes results previously obtained by Jacka, Ansel-Stricker and the authors.

Keywords. arbitrage, martingale, local martingale, equivalent martingale measure, representing measure, risk neutral measure, duality relation, hedging, stochastic integration, mathematical finance.

1991 Mathematics Subject Classification: 90A09, 60G44, 46N10, 47N10, 60H05, 60G40.
\end{abstract}

Part of this research was supported by the European Community Stimulation Plan for Economic Science contract Number SPES-CT91-0089 . 


\section{Introduction.}

In this paper we deal with the change of numéraire problem. Let us assume that a $d$-dimensional process $S$ describes the price of $d$ assets in a fixed chosen currency unit. If e.g. the currency unit is changed, the price process $S$ will be multiplied by the exchange ratio describing the old currency in function of the new one. We shall give examples showing that the No-Arbitrage property of the process $S$ may depend on the choice of numéraire. Such an example was already given in Delbaen Schachermayer [DS1]. The question now arises when the value of an asset or more generally of a portfolio, can be used as a new numéraire without destroying the noarbitrage property. Of course this will depend on the kind of no-arbitrage we use. We will give precise definitions further in the paper but for the moment let us assume (oversimplifying things) that no-arbitrage stands for the existence of an equivalent risk neutral (i.e. for a local martingale) measure.

It turns out that the problem is related to the characterization of those contingent claims that can be hedged. This topic was studied by Jacka [Jk] and Ansel-Stricker [AS]. These authors use the $H^{1}-B M O$ duality. We will give a measure independent characterization in terms of maximal elements of attainable claims. These elements were already used, as a technical device, in our paper Delbaen-Schachermayer [DS2]. The proofs of the theorems below use the results of [DS2] as well as an extension of a duality relation from Delbaen [D].

The technique of a change of numéraire together with the change of the risk neutral measure was used by El Karoui, Geman and Rochet [EGR] and Jamshidian [Jm] to facilitate calculations of prices of contingent claims.

The results of this paper can also be used to build consistent models of exchange rates of currencies. In this case the discounting procedure depends on the currency since the interest rate in different currencies will be different. We refer to Delbaen Shirakawa [DSh] for details.

The rest of this section is devoted to the introduction of the basic notation. Section 2 recalls known facts from arbitrage theory. In section 3 we extend the duality equality and relate it to properties of maximal elements. Section 4 finally contains the main theorem on the change of numéraire and the application to the theory of hedgeable elements.

The setup in this paper is the usual setup in mathematical finance. A probability space $(\Omega, \mathcal{F}, \mathbf{P})$ with a filtration $\left(\mathcal{F}_{t}\right)_{0 \leq t}$ is given. In order to cover the most general case, the time set is supposed to be $\mathbb{R}_{+}$. The filtration is assumed to satisfy the "usual conditions", i.e. it is right continuous and $\mathcal{F}_{0}$ contains all null sets of $\mathcal{F}$. A price process $S$, describing the evolution of the discounted price of $d$ assets, is defined on $\mathbb{R}_{+} \times \Omega$ and takes values in $\mathbb{R}^{\mathrm{d}}$. In order to use the results of Delbaen-Schachermayer [DS2], we suppose that the process $S$ is locally bounded. This assumption is fairly general, in particular it covers the case of continuous price processes. As shown under a wide range of hypotheses, the assumption that $S$ is a semi-martingale follows from arbitrage considerations, see [DS2] and references given there. We can therefore assume that the process $S$ is a semi-martingale. Since it is also locally bounded it is a special semi-martingale. Stochastic integration is used to describe outcomes of investment strategies. When dealing with processes in dimension higher than 1 it is 
understood that vector stochastic integration is used. We refer to Protter $[\mathrm{P}]$ and Jacod $[\mathrm{J}]$ for details on these matters.

The authors want to thank C. Stricker and H. Shirakawa for helpful discussions on the topic. Part of the research was done while the first author was on visit in the University of Tokyo. Discussions with the colleagues and especially with S. Kusuoka, S. Kotani and N. Kunitomo contributed to the development of this paper.

\section{Basic Theorems}

Before proving the main results of the paper we need to recall some definitions and notations introduced in Delbaen-Schachermayer [DS2].

Definition. An $\mathbb{R}^{\mathrm{d}}$-valued predictable process $H$ is called a-admissible if it is $S$ integrable, if $H_{0}=0$, if the stochastic integral satisfies $H \cdot S \geq-a$ and if $(H \cdot S)_{\infty}=$ $\lim _{t \rightarrow \infty}(H \cdot S)_{t}$ exists a.s.. We say that $H$ is admissible if it is a-admissible for some number $a$.

The following notations will be used:

$$
\begin{aligned}
\mathcal{K} & =\left\{(H \cdot S)_{\infty} \mid H \text { is admissible }\right\} \\
\mathcal{K}_{a} & =\left\{(H \cdot S)_{\infty} \mid H \text { is a-admissible }\right\} \\
\mathcal{C}_{0} & =\mathcal{K}-L_{+}^{0} \\
\mathcal{C} & =\mathcal{C}_{0} \cap L^{\infty}
\end{aligned}
$$

The basic theorem in Delbaen-Schachermayer [DS2] uses the concept of No Free Lunch with Vanishing Risk. This is a rather weak form of no-arbitrage-type and it is stated in terms of $L^{\infty}$ convergence. The $N F L V R$ property is therefore independent of the choice of equivalent probability measure. Only the class of negligible sets comes into play.

Definition. We say that the locally bounded semi-martingale $S$ satisfies the No Free Lunch with Vanishing Risk or NFLVR property, with respect to general admissible integrands, if

$$
\overline{\mathcal{C}} \cap L_{+}^{\infty}=\{0\}
$$

where the bar denotes the closure in the supnorm topology of $L^{\infty}$.

The locally bounded semi-martingale $S$ satisfies the No Arbitrage or $N A$ property with respect to general admissible integrands, if

$$
\mathcal{C} \cap L_{+}^{\infty}=\{0\}
$$

The fundamental theorem of asset pricing, as in [DS2] Theorem 1.1, can now be formulated as follows:

Theorem 1. The locally bounded semi-martingale $S$ satisfies the NFLVR property, with respect to general admissible integrands, if and only if there is an equivalent probability measure $\mathbb{Q}$ such that $S$ is a $\mathbb{Q}$-local martingale. In this case the set $\mathcal{C}$ is already weak* (i.e. $\sigma\left(L^{\infty}, L^{1}\right)$ ) closed in $L^{\infty}$.

REMARK. If $\mathbb{Q}$ is an equivalent local martingale measure for $S$ and if $H$ satisfies $H \cdot S \geq-a$ then the result of Ansel-Stricker [AS] shows that $H \cdot S$ is still a local 
martingale and hence, being bounded from below, is a supermartingale. It follows that the limit $(H \cdot S)_{\infty}$ exists a.s. and that $\mathbf{E}_{\mathbb{Q}}\left[(H \cdot S)_{\infty}\right] \leq 0$.

The proof of the fundamental theorem is quite complicated and we cannot repeat it here. The basic idea in [DS2], see lemma 4.4 and the remark following it, is the use of maximal elements in $\mathcal{K}_{1}$. For convenience we give a definition of what we mean by this.

Definition. We say that an element $f \in \mathcal{K}_{a}$ is maximal in $\mathcal{K}_{a}$ if the properties $g \geq f$ a.s. and $g \in \mathcal{K}_{a}$ imply that $g=f$ a.s..

It is easy to see that if $S$ satisfies the No Arbitrage condition then the fact that $f$ is maximal in $\mathcal{K}_{a}$ already implies that $f$ is maximal in $\mathcal{K}_{b}$ for all $b \geq a$ and therefore with the obvious definition also in $\mathcal{K}$. Indeed suppose that $f \in \mathcal{K}_{a}, g=(H \cdot S)_{\infty} \in \mathcal{K}$ and $g \geq f$ a.s., then $g \geq-a$. From lemma 3.5 [DS2] it then follows that $g$ is $a$-admissible and hence the maximality of $f$ in $\mathcal{K}_{a}$ implies that $g=f$ a.s.. An example of an element in $\mathcal{K}_{1}$ that is not maximal will be given below. The $N A$ property with respect to general admissible integrands is now equivalent to the fact that the zero function is maximal in the set $\mathcal{K}$.

In the proof of the fundamental theorem the following intermediate results are shown, again for the (complicated) proof we refer to [DS2] lemma A1.1, lemma 4.3 and the proof of theorem 4.2 :

Theorem 2. If the locally bounded semi-martingale $S$ satisfies the NFLVR property with respect to general admissible integrands, if $\left(f_{n}\right)_{n \geq 1}$ is a sequence in $\mathcal{K}_{1}$, then

(1) there is a sequence of convex combinations $g_{n} \in \operatorname{conv}\left(f_{n}, f_{n+1}, \ldots\right)$ such that $g_{n}$ tends in probability to a function $g$, taking finite values a.s.

(2) there is a maximal element $h$ in $\mathcal{K}_{1}$ such that $h \geq g$ a.s..

Corollary 3. If the locally bounded semi-martingale $S$ satisfies the NFLVR property with respect to general admissible integrands, then the maximal elements of the closure of $\mathcal{K}_{1}$ in $L^{0}$, are in $\mathcal{K}_{1}$.

REMARK. The set $\mathcal{K}_{1}$ is not necessarily closed in the space $L^{0}$. However under the $N F L V R$ property with respect to general admissible integrands, the set $\mathcal{K}_{1}$ and hence its closure are convex and bounded in $L^{0}$. When we define maximal elements of this closure in the obvious way, these maximal elements are already in $\mathcal{K}_{1}$.

The following theorem, in the spirit of [DS2], gives another description of the NFLVR property.

Theorem 4. The locally bounded semi-martingale $S$ satisfies the NFLVR property with respect to general admissible integrands if and only if it satisfies the $N A$ property with respect to general admissible integrands and if there exists a strictly positive local martingale $L$ such that $L_{\infty}>0$ a.s. with $L S$ a local martingale.

ProOf. The necessity is clear. If $\mathbb{Q}$ is an equivalent local martingale measure, then the Radon Nikodym derivative $d \mathbb{Q} / d \mathbf{P}$ defines a strictly positive $\mathbf{P}$-martingale $L$ such that $L S$ is a $\mathbf{P}$ local martingale. Also the process $S$ necessary satisfies the $N A$ property with respect to general admissible integrands. 
The converse is less obvious. We recall from [DS2] corollary 3.8, that it is sufficient to prove that $S$ satisfies $N A$ with respect to general admissible integrands and that the set $\mathcal{K}_{1}$ is bounded in $L^{0}$. If $L$ is a strictly positive local martingale, then the sequence of stopping times defined as

$$
T_{n}=\inf \left\{t \mid L_{t} \geq n\right\}
$$

satisfies $\mathbf{P}\left[T_{n}=\infty\right] \rightarrow 1$ and $L^{T_{n}}$ is a uniformly integrable martingale. These properties follow from the fact that $L$ is a supermartingale and the fact that the jumps of $L$ are necessarily integrable. Also we may and do suppose that $L_{0}=1$. For each $n$ the measure $\mathbb{Q}_{n}$ defined by $d \mathbb{Q}_{n} / d \mathbf{P}=L_{T_{n}}$ is a local martingale measure for the stopped process $S^{T_{n}}$. It follows that the set $\mathcal{K}_{1}$ is bounded when restricted to the event $\left\{T_{n}=\infty\right\}$. Because $\mathbf{P}\left[T_{n}=\infty\right] \rightarrow 1$, this implies that $\mathcal{K}_{1}$ is bounded in $L^{0}$.

The theorem yields the following result, see [DS1] and [DS4] for a different approach and for related results. For details on continuous martingales and Bessel processes we refer to Revuz-Yor [RY].

Corollary 5. If $R$ is the Bessel(3) process, stopped at time 1 and with its natural filtration then $R$ allows arbitrage with respect to general admissible integrands.

Proof. The process $L=\frac{1}{R}$ is a local martingale and from stochastic calculus it follows that it is the only local martingale $X$ such that $X_{0}=1$ and such that $X R$ is a local martingale. If now $\mathbb{Q}$ were a local martingale measure for $R$, then the martingale $X$ defined as $\mathbf{E}_{\mathbf{P}}\left[\frac{d \mathbb{Q}}{d \mathbf{P}} \mid \mathcal{F}_{t}\right]$ satisfies that $X R$ is a local martingale and hence $X=L$. Since $L$ is only a local martingale and not a true martingale we arrive at a contradiction. It follows that $R$ does not have an equivalent local martingale measure. Since it satisfies the second part of the preceding theorem, it cannot satisfy the $N A$ property with respect to general admissible integrands.

REMARK. The element $L_{1}-1$ is not maximal in the set $\mathcal{K}_{1}$ constructed with the process $L$. To see this recall that $\mathbf{E}\left[L_{1}\right]<1$ and that $L_{1}-\mathbf{E}\left[L_{1}\right]$ is by the predictable representation property of $L$, the result of a uniformly integrable martingale of the form $K \cdot L$. It is clear that $(K \cdot L)_{1}=\left(L_{1}-\mathbf{E}\left[L_{1}\right]\right)>L_{1}-1$.

If a locally bounded semi-martingale $S$ satisfies the $N F L V R$ property with respect to general admissible integrands, then the following two non-empty sets will play a role in the theory:

$$
\begin{aligned}
& \mathbf{M}^{e}(\mathbf{P})=\left\{\mathbb{Q} \mid \begin{array}{l}
\mathbb{Q} \text { is equivalent to } \mathbf{P} \\
\text { and the process } S \text { is a } \mathbb{Q} \text {-local martingale }
\end{array}\right\} \\
& \mathbf{M}(\mathbf{P})=\left\{\mathbb{Q} \mid \begin{array}{l}
\mathbb{Q} \text { is absolutely continuous with respect to } \mathbf{P} \\
\text { and the process } S \text { is a } \mathbb{Q} \text {-local martingale }
\end{array}\right\}
\end{aligned}
$$

We identify absolutely continuous measures with their Radon Nikodym derivatives. It is clear that the set $\mathbf{M}^{e}(\mathbf{P})$ is $L^{1}$ dense in $\mathbf{M}(\mathbf{P})$. 


\section{Duality Relation}

In this section we extend the duality formula of Delbaen [D] and Delbaen-Schachermayer [DS2] to the case of unbounded functions. We denote by $\mathcal{C}^{\circ}$ the polar of the cone $\mathcal{C}$, i.e.

$$
\mathcal{C}^{\circ}=\left\{f \mid f \in L^{1}(\mathbf{P}) \text { and for each } h \in \mathcal{C} \text { we have } \mathbf{E}_{\mathbf{P}}[f h] \leq 0\right\} .
$$

Theorem 6. If $S$ is a locally bounded semi-martingale that satisfies the NFLVR property with respect to general admissible integrands then

$$
\mathbf{M}(\mathbf{P})=\mathcal{C}^{\circ} \bigcap\{\mathbb{Q} \mid \mathbb{Q} \text { probability measure, } \mathbb{Q} \ll \mathbf{P}\}
$$

Proof. If $\mathbb{Q} \in \mathbf{M}(\mathbf{P})$ then for each admissible integrand $H$ we have, by the Ansel-Stricker theorem, $[\mathrm{AS}]$, that $H \cdot S$ is a $\mathbb{Q}$-local martingale and hence it is a supermartingale. Therefore $\mathbf{E}_{\mathbb{Q}}[f] \leq 0$ for each $f \in \mathcal{K}$. The same inequality pertains for elements of $\mathcal{C}$.

Conversely if $\mathbb{Q}$ is a probability measure in $\mathcal{C}^{\circ}$ then $S$ will be a $\mathbb{Q}$-local martingale. Indeed take $T_{n}$ an increasing sequence of stopping times, $T_{n} \uparrow \infty$, such that each $S^{T_{n}}$ is bounded. For each $s<t$ and each $A \in \mathcal{F}_{s}$ we have that $\mathbf{1}_{A}\left(S_{t}^{T_{n}}-S_{s}^{T_{n}}\right)$ is in $\mathcal{C}$ and hence we have $\mathbf{E}_{\mathbb{Q}}\left[\mathbf{1}_{A}\left(S_{t}^{T_{n}}-S_{s}^{T_{n}}\right)\right] \leq 0$. Replacing $\mathbf{1}_{A}$ by $-\mathbf{1}_{A}$ gives that $\mathbf{E}_{\mathbb{Q}}\left[\mathbf{1}_{A}\left(S_{t}^{T_{n}}-S_{s}^{T_{n}}\right)\right]=0$. These equalities show that $S$ is a $\mathbb{Q}$-local martingale.

Corollary 7. Suppose that the locally bounded semi-martingale $S$ satisfies the NFLVR property with respect to general admissible integrands. The set $\mathbf{M}(\mathbf{P})$ is then closed in $L^{1}(\mathbf{P})$.

We remark that this is essentially a consequence of the local boundedness of $S$. It is easy to give counter-examples in the general case.

Theorem 8. If the locally bounded semi-martingale $S$ satisfies the NFLVR property with respect to general admissible integrands, then for bounded elements $f$ in $L^{\infty}$ we have that

$$
\begin{aligned}
\sup _{\mathbb{Q} \in \mathbf{M}^{e}(\mathbf{P})} \mathbf{E}_{\mathbb{Q}}[f] & =\sup _{\mathbb{Q} \in \mathbf{M}(\mathbf{P})} \mathbf{E}_{\mathbb{Q}}[f] \\
& =\inf \{x \mid \exists h \in \mathcal{C} \quad x+h \geq f\} \\
& =\inf \{x \mid \exists h \in \mathcal{C} \quad x+h=f\} \\
& =\inf \{x \mid(f-x) \in \mathcal{C}\} \\
& =\inf \{x \mid \exists h \in \mathcal{K} \quad x+h \geq f\}
\end{aligned}
$$

Furthermore all infima are minima.

Proof. The proof of this theorem is an application of the previous theorem and duality theory

The first equality is almost trivial since $\mathbf{M}^{e}(\mathbf{P})$ is dense in $\mathbf{M}(\mathbf{P})$ for the norm topology of $L^{1}(\mathbf{P})$. Suppose that $f \leq x+h$ where $h \in \mathcal{C}$. It follows from the 
preceding theorem that for all $\mathbb{Q} \in \mathbf{M}(\mathbf{P})$ we have that $\mathbf{E}_{\mathbb{Q}}[f] \leq x+\mathbf{E}_{\mathbb{Q}}[h] \leq x$. It is therefore obvious that

$$
\sup _{\mathbb{Q} \in \mathbf{M}(\mathbf{P})} \mathbf{E}_{\mathbb{Q}}[f] \leq \inf \{x \mid \exists h \in \mathcal{C} \quad x+h \geq f\} .
$$

The converse inequality is proved using the Hahn-Banach theorem and the fact that the set $\mathcal{C}$ is weak* closed, see Theorem 1 above. Let $z$ be a real number such that

$$
z<\inf \{x \mid \exists h \in \mathcal{C} \quad x+h \geq f\} .
$$

We have that $f-z \notin \mathcal{C}$. By the Hahn-Banach theorem there is a weak* continuous functional on $L^{\infty}$, denoted by the corresponding measure $\mathbb{Q}$, such that for all $h \in \mathcal{C}$ we have

$$
\int(f-z) d \mathbb{Q}>\int h d \mathbb{Q} .
$$

Since $\mathcal{C}$ is a cone containing $-L_{+}^{\infty}$, this necessarily implies that for all $h \in \mathcal{C}$ we have

$$
0 \geq \int h d \mathbb{Q} \text { and that } \int(f-z) d \mathbb{Q}>0
$$

We deduce that $\mathbb{Q}$ is necessarily positive and we may therefore suppose that $\mathbb{Q}$ is normalized in such a way that $\mathbb{Q}(\Omega)=1$. In that case $\mathbb{Q}$ is a probability measure, is an element of $\mathcal{C}^{\circ}$ and hence an element of $\mathbf{M}(\mathbf{P})$. But then the second inequality shows that $\mathbf{E}_{\mathbb{Q}}[f]>z$. We obtain that

$$
\sup _{\mathbb{Q} \in \mathbf{M}(\mathbf{P})} \mathbf{E}_{\mathbb{Q}}[f] \geq \inf \{x \mid \exists h \in \mathcal{C} \quad x+h \geq f\} .
$$

and this ends the proof of the equalities. The fact that all infima are minima is an easy consequence of the closedness of $\mathcal{C}$ for the norm topology of $L^{\infty}$. Indeed, the set $\{x \mid(f-x) \in \mathcal{C}\}$ is closed.

We will now generalize the preceding equalities to arbitrary positive functions. The proof relies on the special properties of the sets $\mathcal{C}$ and $\mathcal{K}$.

Theorem 9. Suppose that the locally bounded martingale $S$ satisfies the NFLVR property with respect to general admissible integrands. If $f \geq 0$, or more generally if $f$ is bounded below by a constant, then

$$
\begin{aligned}
\sup _{\mathbb{Q} \in \mathbf{M}^{e}(\mathbf{P})} \mathbf{E}_{\mathbb{Q}}[f]= & \sup _{\mathbb{Q} \in \mathbf{M}(\mathbf{P})} \mathbf{E}_{\mathbb{Q}}[f] \\
= & \inf \{x \mid \exists h \in \mathcal{K} \quad x+h \geq f\} \\
& \text { and when the expression is finite } \\
= & \min \{x \mid \exists h \in \mathcal{K} \quad x+h \geq f\} .
\end{aligned}
$$

Proof. We suppose that $f \geq 0$. The first equality follows again from the density of $\mathbf{M}^{e}(\mathbf{P})$ in the set $\mathbf{M}(\mathbf{P})$ and Fatou's lemma. The left hand side is smaller than 
the right hand side exactly as in the proof of the previous theorem. We remark that this already implies that we have equality as soon as $\sup _{\mathbb{Q} \in \mathbf{M}^{e}(\mathbf{P})} \mathbf{E}_{\mathbb{Q}}[f]=\infty$. Let now $z$ be a real number such that $z>\sup _{\mathbb{Q} \in \mathbf{M}^{e}(\mathbf{P})} \mathbf{E}_{\mathbb{Q}}[f]$. For all natural numbers we therefore have that $z>\sup _{\mathbb{Q} \in \mathbf{M}^{e}(\mathbf{P})} \mathbf{E}_{\mathbb{Q}}[f \wedge n]$. The theorem for bounded functions now implies the existence of $h_{n} \in \mathcal{K}$ and $0 \leq x_{n}<z$ such that $f \wedge n \leq x_{n}+h_{n}$. We may extract subsequences and suppose that the bounded sequence $x_{n}$ converges to a real number $x \leq z$. The functions $h_{n}$ are bigger than $-x_{n}$ and therefore the result of an $x_{n}$ and hence a $z$-admissible strategy $H^{n}$. The sequence of functions $h_{n}$ is in $\mathcal{K}_{z}$, a bounded convex set of $L^{0}(\mathbf{P})$. Using lemma A1.1 from [DS2] we may take convex combinations of $h_{n}$ that converge almost everywhere to a function $h$. We still have that $h+x \geq f$. The properties of $\mathcal{K}_{z}$ listed above (see Theorem 2, (2)), imply that there is an element $g \in \mathcal{K}_{z}$ such that $g \geq h$. This element clearly satisfies $x+g \geq f$ and hence we obtain

$$
z \geq \inf \{x \mid \exists h \in \mathcal{K} \quad x+h \geq f\}
$$

We therefore see that

$$
\sup _{\mathbb{Q} \in \mathbf{M}^{e}(\mathbf{P})} \mathbf{E}_{\mathbb{Q}}[f]=\sup _{\mathbb{Q} \in \mathbf{M}(\mathbf{P})} \mathbf{E}_{\mathbb{Q}}[f]=\inf \{x \mid \exists h \in \mathcal{K} \quad x+h \geq f\}
$$

To see that the infimum is a minimum we take a sequence $x_{n}$ tending to the infimum and a corresponding sequence of outcomes $h_{n}$. We can apply the same reasoning to see that the infimum is attained.

Corollary 10. Suppose that the locally bounded semi-martingale $S$ satisfies the NFLVR-property with respect to general admissible integrands. If $f \geq 0$ and if $x=\sup _{\mathbb{Q} \in \mathbf{M}^{e}(\mathbf{P})} \mathbf{E}_{\mathbb{Q}}[f]<\infty$, then there is a maximal element $g \in \mathcal{K}$ such that $f \leq x+g$.

PROOF. This follows from the proof of the theorem.

\section{Hedging and Change of Numéraire}

Before we give a martingale characterization of maximal elements of $\mathcal{K}$, we first study the $N A$ property under the change of numéraire. Since we want to apply it in a fairly general setting, we will work with an abstract $\mathbb{R}^{\mathrm{d}}$ valued semi-martingale $W$. In this section we do not even require the semi-martingale to be locally bounded. When we change the numéraire from the constant 1 into the process $V$ we will have to rescale the process $W$. The best way to do this is to introduce the $(d+2)$-dimensional process $(W, 1, V)$. The constant 1 , which corresponds to the original numéraire was added, because under the new numéraire $V$, this will not be constant anymore but will be replaced by $\frac{1}{V}$. On the other hand, the process $V$ will be replaced by 1 . By adding this constant process, we obtain more symmetry. Under the new numéraire the system is described by the process $\left(\frac{W}{V}, \frac{1}{V}, 1\right)$. Before proving the change of numéraire theorem, a theorem that relates the $N A$ property of both systems, let us give an example of what happens in a discrete time setting and when $d=0$, the simplest possible case. 
ExAMPLE. The semi-martingale $V$ which describes the price of the new numéraire (in terms of the old one) is supposed to satisfy $V_{0}=1$, a pure normalization assumption, $V_{t}>0$, a.s. and $\lim _{t \rightarrow \infty} V_{t}=V_{\infty}$ exists a.s. and is strictly positive a.s.. Note that the symmetry in these assumptions if we pass from $V$ to $\frac{1}{V}$, i.e. they are invariant whether we consider the new numéraire in terms of the old one or vice versa. The process is driven by a sequence of independent identically distributed Bernoulli variables $\left(\varepsilon_{n}\right)_{n \geq 1}$. They are such that $\mathbf{P}\left[\varepsilon_{n}=1\right]=\mathbf{P}\left[\varepsilon_{n}=-1\right]=1 / 2$. To facilitate the writing, we call the two currencies ECU and \$. The process $V$ describes the value of the $\$$ in terms of the ECU. Let us now fix $\alpha$ such that $0<\alpha<1$. At time $n=0$, we require that $V_{0}=1$. Let us suppose that $V_{n-1}$ is already defined. If the Bernoulli variable $\varepsilon_{n}=1$ then we put $V_{n}=\alpha$. If $\varepsilon_{n}=-1$, then we put $V_{n}=2 V_{n-1}-\alpha$. In such a way the process $V$ remains strictly positive, in fact greater than $\alpha$, it becomes eventually equal to $\alpha$ and the limit $V_{\infty}=\alpha$ therefore exists. The process $V$ is also a non uniformly integrable martingale with respect to the measure $\mathbf{P}$. Remark that once the process hits the level $\alpha$ it remains at that level forever. In economic terms we may say that an investment in $\$$ seems to be a fair game, since $V$ is a martingale, but that at the end it was not a good choice. Indeed, since $\alpha<1$, the investment is, in the long run, a losing one. An economic agent might try to get a profit out of it by selling short the $\$$. But here is an obstruction. Indeed by going short on $\$$, the ECU investor will realize that he is using a non admissible strategy. Therefore she will not be able to take advantage of this special situation. A $\$$ investor on the contrary is able to buy ECU at an initial price of $1 \$$ and then in the long run sell this ECU for $1 / \alpha$, making arbitrage profits! As a last point let us observe that the 0 -variable dominates the outcome $V_{\infty}-1=\alpha-1$ and hence the variable $V_{\infty}-1$ is not maximal. The example is simple but it has all the features that appear in greater generality in the theorem.

Theorem 11. Let $W$ be a semi-martingale, taking values in $\mathbb{R}^{\mathrm{d}}$. Let $V$ be a strictly positive semi-martingale such that $V_{\infty}=\lim _{t \rightarrow \infty} V_{t}$ exists and is strictly positive a.s. The semi-martingale $X$ is the $d+2$ dimensional process $X=(W, 1, V)$. The process $Z$ defined as $Z=\left(\frac{W}{V}, \frac{1}{V}, 1\right)$ is a $d+2$ dimensional semi-martingale. It satisfies the $N A$ property with respect to general admissible integrands if and only if $V_{\infty}-1$ is maximal in the set of outcomes of 1-admissible integrands for $X$.

Proof. Using the symmetry between the processes $X$ and $Z$ we first reformulate the statement of the theorem. We can regard the process $X$ as obtained from $Z$ by dividing it by the process $\frac{1}{V}$. The process $\frac{1}{V}$ is also strictly positive and at infinity its limit exists a.s. and is still strictly positive. If we change the role of $X$ and $Z$, resp. $V$ and $\frac{1}{V}$, we see that the proof of the theorem is equivalent to the proof of the following two statements

(1) If $X$ satisfies the $N A$ property with respect to general admissible integrands then $\frac{1}{V_{\infty}}-1$ is maximal in the set of outcomes of 1 -admissible integrands for $Z$.

(2) If $Z$ permits arbitrage with respect to general admissible integrands then $V_{\infty}-1$ is not maximal in the set of outcomes of 1-admissible integrands for $X$. 
The proof depends on the following calculation from vector stochastic calculus. From $X=V Z$ we deduce that

$$
d X_{t}=d V_{t} Z_{t-}+V_{t-} d Z_{t}+d[V, Z]_{t}
$$

If $K$ is a $(d+2)$-dimensional predictable process that is a 1-admissible integrand for the system $Z=\left(\frac{W}{V}, \frac{1}{V}, 1\right)$ then we let $Y=(1+K \cdot Z) V$. Remark that $Y$ is a process that describes a portfolio obtained by using an investment described by the system $Z$ that afterwards is converted, through the change of numéraire $V$, into values that fit in the system $X$. We have that

$$
d Y_{t}=d V_{t}\left(1+(K \cdot Z)_{t-}\right)+V_{t-} K_{t} d Z_{t}+K_{t} d[V, Z]_{t}
$$

Using the expression for $d X$ we may convert this into

$$
d V_{t}\left(1+(K \cdot Z)_{t-}\right)+K_{t} d X_{t}-d V_{t} K_{t} Z_{t-}
$$

which is of the form

$$
d Y_{t}=L_{t} d X_{t}
$$

for some $d+2$ dimensional predictable and $X$-integrable process $L$. Since $K$ was 1-admissible for $Z$, we have that $Y$ is positive and therefore $L$ is 1-admissible for $X$. We now apply the above equality in two different cases. To prove (1) we suppose that $\frac{1}{V_{\infty}}-1$ is not maximal. Take $K$ a 1 -admissible integrand for $Z$ such that the limit at infinity exists and such that $1+(K \cdot Z)_{\infty} \geq \frac{1}{V_{\infty}}$, with strict inequality on a non-negligible set. In that case we have that $Y_{\infty}-1=(L \cdot X)_{\infty}$ is non-negative and strictly positive on a non negligible set. This should produce arbitrage for $X$.

The second part is proved in a similar way. Suppose that $Z$ allows arbitrage and that $K$ is the 1 -admissible integrand responsible for it. The outcome $Y_{\infty}-1$ is now greater than $V_{\infty}-1$, with strict inequality on a non negligible set. A contradiction to its maximality.

Corollary 12. Using the same notation as in the theorem we see that $X$ satisfies the $N A$ property with respect to general admissible integrands and $V_{\infty}-1$ is maximal "for $X$ " if and only if $Z$ satisfies the $N A$ property with respect to general admissible integrands and $\frac{1}{V_{\infty}}-1$ is maximal "for $Z$ ".

ProOF. This is a straightforward application of the previous theorem. The only difference lies in the statement that $V_{\infty}-1$ is maximal in the set of all outcomes of admissible integrand and not just in the set of outcomes of 1-admissible integrands. If $X$ satisfies $N A$ and $V_{\infty}-1$ is maximal then we can apply both parts of the theorem. In this case we know, from section 2 , that $f=\frac{1}{V_{\infty}}-1$ is maximal in the set of outcomes of all admissible integrands. This proves the if statement. The only if part is the same statement as the if part because $X$ is obtained from $Z$ by multiplying with $V^{-1}$.

We can now apply the above reasoning to the original setting of this paper. Given a locally bounded semi-martingale $S$ that satisfies the $N F L V R$ property with respect 
to general admissible integrands, we use a process of the form $V=1+H \cdot S$ for the new numéraire. If $H$ is admissible and $V_{\infty}>0$ a.s., then we can apply the previous theorem. In this case we certainly have that the system $(S, 1, V)$ has the $N A$ property with respect to general admissible integrands. With the assumption that $\mathbf{P}$ was a local martingale measure for $S$, the system $(S, 1, V)$ becomes in fact a local martingale for P. The previous theorem then yields

Theorem 13. Suppose that $S$ is a locally bounded semi-martingale that satisfies the NFLVR property with respect to general admissible integrands. Suppose that $H$ is admissible and that the process $V=1+(H \cdot S)$ satisfies $f=V_{\infty}=1+(H \cdot S)_{\infty}>0$ a.s.. Then the following are equivalent:

(1) $(H \cdot S)_{\infty}$ is maximal in the set $\mathcal{K}$.

(2) The process $\tilde{S}=\left(\frac{S}{V}, \frac{1}{V}\right)$ satisfies $N A$ with respect to general admissible integrands.

(3) There is $\mathbb{Q} \in \mathbf{M}^{e}(\mathbf{P})$ such that $H \cdot S$ is a $\mathbb{Q}$-uniformly integrable martingale. If $V^{-1}$ is locally bounded then these statements are equivalent to :

(4) The process $\tilde{S}$ has an equivalent local martingale measure.

REMARK. We conjecture that the assumption that $V^{-1}$ is locally bounded can be removed.

ProOF. 1 and 2 are equivalent

Since $S$ satisfies the NFLVR property with respect to general admissible integrands, there is an equivalent local martingale measure, $\mathbb{Q}$ for $S$. Because the stochastic integral $H \cdot S$ is bounded below, the theorem of Ansel-Stricker, see [AS], implies that it, and hence also $V$, is a local martingale. Since the final value $V_{\infty}$ of $V$ is strictly positive, the result in Dellacherie-Meyer [DM] Th 17, p. 85 implies that the process $V$ is bounded away from zero a.s.. We can now apply Theorem 11 to see that (1) and (2) are already equivalent.

\section{1 implies 4}

In case $V^{-1}$ is locally bounded we have that $\tilde{S}$ is also locally bounded. It has the $N A$ property and the product $V \tilde{S}$ is a local martingale. Therefore the process has the $N F L V R$ property and by Theorem 1 and Theorem 4 it has an equivalent local martingale measure.

\section{1 and/or 2 imply 3}

Now we apply the statement that (1) implies (4) on the process $V^{\prime}=\frac{1}{2}(1+V)$. This process is defined using $H / 2$ instead of $H$. It has the advantage that $\frac{1}{V^{\prime}}$ is bounded. Let $\tilde{\mathbb{Q}}$ be an equivalent local martingale measure for $\left(\frac{S}{V^{\prime}}, \frac{1}{V^{\prime}}\right)$. Since the last coordinate $X=\frac{1}{V^{\prime}}$ is bounded and is a $\tilde{\mathbb{Q}}$-local martingale it is a strictly positive bounded martingale, starting at 1 . When we define the probability measure $\mathbb{Q}$ by $d \mathbb{Q}=X_{\infty} d \tilde{\mathbb{Q}}$, we obtain that $S=\frac{S}{V^{\prime}} V^{\prime}$ is a $\mathbb{Q}$-local martingale and $V^{\prime}$ is a $\mathbb{Q}$ uniformly integrable martingale. This implies that $H \cdot S$ is a $\mathbb{Q}$-uniformly integrable martingale. The proof that (1) and/or (2) implies (3) is complete.

\section{3 implies 1}


If $H \cdot S$ is a $\mathbb{Q}$-uniformly integrable martingale for some $\mathbb{Q} \in \mathbf{M}^{e}(\mathbf{P})$ then $(H \cdot S)_{\infty}$ is necessarily maximal. Indeed if say $(K \cdot S)_{\infty} \geq(H \cdot S)_{\infty}$ fro some admissible $K$, then by taking expectations with respect to $\mathbb{Q}$, applying the supermartingale property of $K \cdot S$ and the martingale property of $(H \cdot S)$ we see

$$
0=\mathbf{E}_{\mathbb{Q}}\left[(H \cdot S)_{\infty}\right] \leq \mathbf{E}_{\mathbb{Q}}\left[(K \cdot S)_{\infty}\right] \leq 0 .
$$

It follows that $\mathbf{E}_{\mathbb{Q}}\left[(K \cdot S)_{\infty}\right]=0$ and $(H \cdot S)_{\infty}=(K \cdot S)_{\infty}$. This completes the proof that (3) implies (1).

\section{4 implies 2}

Since the existence of an equivalent local martingale measure implies the $N A$ property with respect to general admissible integrands, this is trivial.

Corollary 14. If the locally bounded semi-martingale $S$ satisfies the NFLVR property with respect to general admissible integrands then for an admissible integrand $H$ the following are equivalent:

(1) $(H \cdot S)_{\infty}$ is maximal in $\mathcal{K}$

(2) there is $\mathbb{Q} \in \mathbf{M}^{e}(\mathbf{P})$ such that $\mathbf{E}_{\mathbb{Q}}\left[(H \cdot S)_{\infty}\right]=0$

(3) there is $\mathbb{Q} \in \mathbf{M}^{e}(\mathbf{P})$ such that $H \cdot S$ is a $\mathbb{Q}$-uniformly integrable martingale

The theorem also allows us to give a characterization of strict local martingales as studied by Elworthy, Li and Yor, [ELY]. They define a strict local martingale as a local martingale that is not a uniformly integrable martingale.

Corollary 15. Let $S=L$ be a strictly positive locally bounded local martingale such that $L_{\infty}>0$ a.s.. Let

$$
\mathbf{M}^{e}(\mathbf{P})=\left\{\mathbb{Q} \mid \begin{array}{l}
\mathbb{Q} \text { is equivalent to } \mathbf{P} \\
\text { and the process } L \text { is a } \mathbb{Q} \text {-local martingale }
\end{array}\right\}
$$

The process $\frac{1}{L}$ satisfies the $N A$ property with respect to general admissible integrands if and only if $L$ is a uniformly integrable martingale for some $\mathbb{Q}$ in $\mathbf{M}^{e}(\mathbf{P})$.

REMARK. From Schachermayer [S] (see Delbaen Schachermayer [DS4] for an easier example) it follows that under the assumptions of the corollary, the process $L$ need not be a uniformly integrable martingale under all elements of $\mathbf{M}^{e}(\mathbf{P})$.

REMARK. In the case that $R=\frac{1}{L^{1}}$ equals the Bessel(3) process with its natural filtration, stopped at time 1 , we have that $L^{1}$ is a local martingale for $\mathbf{P}$. This is the only martingale measure and hence we deduce that $R$ has arbitrage with respect to general admissible integrands. The preceding corollary is a generalization of this phenomenon to the case that $\mathbf{M}^{e}(\mathbf{P})$ is not a singleton, see also section 2 .

Definition. If $S$ is a locally bounded semi-martingale that satisfies the NFLVR property with respect to general admissible integrands, then we say that a positive random variable (or contingent claim) $f$ can be hedged if there is $x \in \mathbb{R}$ and a maximal element $h \in \mathcal{K}$ such that $f=x+h$.

There is a good reason to require the use of maximal elements. If $h$ is not maximal then there is a maximal element $g \in \mathcal{K}, g \neq h$ such that $g \geq h$. An investor who 
would try to hedge $f$ by using an admissible strategy, would be better off to use a strategy that gives her the outcome $g$ instead of $h$. Starting with the same initial investment $x$, she will obtain something better than $f$ and since $g>h$ on a set of positive measure, she will be strictly better off in some cases. In such a case the contingent claim $f$ is not the result of a good optimal hedging policy.

The following theorem is due to Ansel-Stricker [AS] and, independently, to Jacka [Jk]. They proved it using $H^{1}-B M O$ duality. We shall see that it is also a consequence of the characterization of maximal elements.

Theorem 16. If $S$ is a locally bounded semi-martingale that satisfies the NFLVR property with respect to general admissible integrands then for a random variable $f \geq 0$, the following are equivalent

(1) $f$ can be hedged

(2) there is $\mathbb{Q}$ in $\mathbf{M}^{e}(\mathbf{P})$ such that

$$
\mathbf{E}_{\mathbb{Q}}[f]=\sup \left\{\mathbf{E}_{\mathbf{R}}[f] \mid \mathbf{R} \in \mathbf{M}^{e}(\mathbf{P})\right\}<\infty
$$

PROOF.

(1) implies (2)

If $f$ can be hedged, then there is an admissible strategy $H$ and a real number $x$, such that $f=x+(H \cdot S)_{\infty}$ and $H \cdot S$ is a uniformly integrable martingale for some $\mathbb{Q} \in \mathbf{M}^{e}(\mathbf{P})$. For all $\mathbf{R} \in \mathbf{M}^{e}(\mathbf{P})$ we have that $H \cdot S$ is a supermartingale and hence $\mathbf{E}_{\mathbf{R}}[f] \leq x=\mathbf{E}_{\mathbb{Q}}[f]$.

(2) implies (1)

If we have $\mathbf{E}_{\mathbb{Q}}[f]=\sup \left\{\mathbf{E}_{\mathbf{R}}[f] \mid \mathbf{R} \in \mathbf{M}^{e}(\mathbf{P})\right\}<\infty$, then clearly we have that

$$
x=\mathbf{E}_{\mathbb{Q}}[f]=\min \{z \mid \exists h \in \mathcal{K} \text { such that } z+h \geq f\}<\infty
$$

The duality relation of section 3 now implies that there is an admissible integrand $H$ such that $f \leq x+(H \cdot S)_{\infty}$. Since $H \cdot S$ is a supermartingale for $\mathbb{Q}$ we have that

$$
x=\mathbf{E}_{\mathbb{Q}}[f] \leq x+\mathbf{E}_{\mathbb{Q}}\left[(H \cdot S)_{\infty}\right] \leq x
$$

and hence $\mathbf{E}_{\mathbb{Q}}\left[(H \cdot S)_{\infty}\right]=0$. This implies that $f=x+(H \cdot S)_{\infty}$ and that $H \cdot S$ is uniformly integrable for $\mathbb{Q}$. Therefore $(H \cdot S)_{\infty}$ is maximal in $\mathcal{K}$.

\section{References}

[AS] J.P. Ansel and C. Stricker, Couverture des actifs contingents, preprint (1992).

[D] F. Delbaen, Representing Martingale Measures when Asset Prices are Continuous and Bounded, Mathematical Finance 2 (1992), 107-130.

[DS1] F. Delbaen and W. Schachermayer, Arbitrage and Free Lunch with Bounded Risk for unbounded continuous processes, Mathematical Finance (1994).

[DS2] F. Delbaen and W. Schachermayer, A General Version of the Fundamental Theorem of Asset Pricing, Mathematische Annalen (1994). 
[DS3] F. Delbaen and W. Schachermayer, Arbitrage possibilities in Bessel processes and their relations to local martingales, forthcoming (1994).

[DS4] F. Delbaen and W. Schachermayer, A Simple Example of Two Non Uniformly Integrable Continuous Martingales whose product is a Uniformly Integrable Martingale, forthcoming (1994).

[DSh] F. Delbaen and H. Shirakawa, An Arbitrage Free Model of Exchange Rates, forthcoming (1994).

[DM] C. Dellacherie and P. A. Meyer, Probabilités et Potentiel, Chap.V-VIII., Hermann, Paris, 1980.

[EGR] N. El Karoui, H. Geman and Rochet, Changes of Numéraire, Changes of Probability Measure and Option Pricing, Advances in Applied Probability (1994).

[ELY] D. Elworthy, X.-M. Li and M. Yor, The Importance of Strict Local Martingales, forthcoming (1993).

[J] J. Jacod, Calcul Stochastique et problèmes de martingales, Springer Verlag, Berlin, Heidelberg, New York, 1979.

[Jk] S. Jacka, A Martingale Representation Result and an Application to Incomplete Financial Markets, Mathematical Finance 2 (1992), 239-250.

[Jm] F. Jamshidian, Pricing of Contingent Claims in the One Factor Term Structure Model, Merrill Lynch Capital Markets, Research Paper (1987).

[P] Ph. Protter, Stochastic Integration and Differential Equations, a New Approach, Springer Verlag, Berlin, Heidelberg, New York, 1990.

[RY] D. Revuz and M. Yor, Continuous Martingales and Brownian Motion, Springer Verlag, Berlin Heidelberg New York., 1991.

[S] W. Schachermayer, A Counterexample to Several Problems in the Theory of Asset Pricing, Mathematical Finance 3 (1993), 217-230. 